\title{
Pronóstico del Precio del Petróleo mediante Redes Neuronales Artificiales
}

\author{
Fernando Villada(1), Daniel Arroyave(1) y Melissa Villada(2) \\ (1) Fac. de Ingeniería, Depto Ing. Eléctrica, Universidad de Antioquia, Calle 67 No. 53-108, Oficina 19-407, \\ Medellín-Colombia (e-mail: fvillada@udea.edu.co) \\ (2) Escuela de Administración, Universidad Eafit, Carrera 49 No. 7 sur 50, Medellín- Colombia.
}

Recibido Oct. 31, 2013; Aceptado Dic. 30, 2013; Versión final recibida Ene. 24, 2014

\begin{abstract}
Resumen
Este trabajo propone un modelo basado en redes neuronales artificiales para el pronóstico del precio internacional del petróleo. Para el desarrollo del modelo se usan datos de precios de la literatura para el petróleo de referencia WTI (West Texas Intermediate) transado principalmente en la Bolsa Mercantil de Nueva York. Se utilizan cuatro estructuras de redes incluyendo como entradas la serie de precios diarios en la primera, la serie de precios más el índice del dólar estadounidense DXY en la segunda, la serie de precios más el índice S\&P500 en la tercera y la serie de precios más los índices DXY y S\&P500 en la cuarta. Se prueban diferentes configuraciones de redes neuronales utilizando una serie de seis meses, donde los datos de los primeros cinco se utilizan para entrenamiento dejando el último mes para verificar las capacidades predictivas del modelo. Se analiza también el efecto de incluir el grado de aversión al riesgo de los inversionistas por medio de los índices DXY y S\&P500 como variables adicionales de entrada. Los resultados muestran un buen comportamiento de las redes neuronales con bajos errores en aprendizaje y en predicción
\end{abstract}

Palabras clave: mercado del petróleo, redes neuronales artificiales, pronóstico de precios

\section{Oil Price Forecast using Artificial Neural Networks}

\begin{abstract}
An artificial neural network model to forecast oil international price is proposed in this work. To develop the model, price data taken from the literature for the WTI reference oil (West Texas Intermediate) traded mainly in New York Mercantile Exchange are used. Four network structures, including the daily price series in the first one, the price series plus the dollar index DXY in the second one, the price series plus the S\&P500 index in the third one and the price series plus the DXY and S\&P500 indexes in the fourth one are used. Different neural networks configurations are analyzed using a series of six months, where data for five months are used for training patterns and the next month is left for testing the predictive capabilities of the model. The effect of including investors risk aversion using the DXY and S\&P500 indexes as alternative input patterns is also analyzed. The results show good performance of the neural networks both during learning and prediction.
\end{abstract}

Keywords: oil exchange market, artificial neural networks, price forecasting 


\section{INTRODUCCIÓN}

El petróleo es el bien básico más negociado en todo el mundo, no sólo en términos de volumen sino también de montos de dinero. Su consumo por lo general se da en lugares diferentes a los de su extracción, destacándose Estados Unidos como el mayor consumidor bruto y neto de petróleo en todo el mundo; a pesar de ello, la región con mayor importación neta es la asiática. Usualmente, los precios del petróleo son determinados por la interacción entre las fuerzas de oferta y demanda, sin embargo, presentan alta volatilidad especialmente en periodos de inestabilidad política y guerras en los países del medio oriente, crecimiento o recesión económica y las decisiones sobre producción de la organización de países exportadores de petróleo (OPEC). Los factores más influyentes en el precio del petróleo son los costos de extracción, transporte, transformación y manejo de inventarios, las expectativas de los agentes, las barreras o problemas geopolíticos en el medio oriente y las decisiones de producción de la organización de la OPEC.

Las exportaciones e importaciones de petróleo juegan un papel importante en las cuentas de la balanza de pagos de los países, puesto que en el momento en el que los ingresos por compra y venta de petróleo entran al mercado cambiario, la cuenta corriente se ve directamente afectada. Por esta razón se hace cada vez más pertinente el estudio de modelos que permitan pronosticar el comportamiento del precio del petróleo de tal forma que se constituyan en una guía que oriente planeación macroeconómica de los diferentes países y a la vez oriente la estrategia a seguir por los inversionistas participantes en el mercado.

Diversos modelos matemáticos han sido publicados para el estudio del comportamiento del mercado del petróleo. Zamani (2004) presenta un modelo autorregresivo para predecir el precio spot del petróleo WTI en función del nivel de inventarios de los países miembros de la OCDE (Organización para la Cooperación y el Desarrollo Económico), la demanda de los países no-OCDE y el nivel de producción de la OPEC; los resultados del pronóstico son alentadores y plantean su utilidad para los diseñadores de política económica y la OPEC en la determinación del nivel de producción. Robles-Agudo y Vásquez-Román (2008) proponen un modelo matemático para optimizar la planeación de la producción de gas y petróleo en varios pozos obteniendo resultados satisfactorios al poder involucrar diversas restricciones de presión en tuberías y de demanda. Unas de las técnicas matemáticas utilizadas para abordar el estudio del comportamiento de los mercados han sido los modelos multivariantes y univariantes, pero presentan deficiencias cuando se trata de realizar predicciones fuera de la muestra (Meese y Roese, 1991). En un trabajo más reciente, Ghoshray y Johnson (2010) aplicaron modelos univariados al pronóstico de precios del petróleo, el gas y el carbón detectando roturas estructurales en las tendencias lo que sugiere el efecto de estos cambios de la economía global en el comportamiento de los precios de los energéticos en el largo plazo.

La revisión detallada de la literatura disponible sobre modelos econométricos para el pronóstico del precio del petróleo fue realizada por Frey et al. (2009). En este trabajo distinguen tres categorías de modelos econométricos: modelos de series de tiempo explotando las propiedades estadísticas de los datos, modelos financieros basados en la relación entre los precios spot y futuro y modelos estructurales describiendo el efecto de los factores económicos sobre el valor futuro de los precios. El trabajo concluye la imposibilidad de identificar el modelo econométrico más adecuado para el pronóstico ya que todos han mostrado un desempeño satisfactorio dependiendo de la información utilizada, el escenario de tiempo utilizado, las condiciones económicas del momento y las mediciones de error utilizadas.

Un elemento adverso para el mercado del petróleo con su respectiva influencia en el precio lo constituye la creciente preocupación mundial por el calentamiento global. En un trabajo reciente, Espíndola y Valderrama (2012) analizan el concepto de huella de carbono y su relación con los gases de efecto invernadero y el calentamiento global, enfatizando en la necesidad de avanzar en la difusión de bases conceptuales y adoptar una estrategia para el logro de un crecimiento global sostenible y la sustentabilidad de las organizaciones.

Estudios posteriores han demostrado que la presencia de dinámicas no lineales hacen necesario el empleo de modelos más sofisticados. En este sentido, Kisswani y Nusair (2013) no encontraron evidencia de estacionariedad en las series de precios del petróleo (Brent, Dubai y WTI) lo cual es atribuido a la presencia de no linealidades en el comportamiento del precio del petróleo. Algunas posibles causas de estas nolinealidades pueden ser las barreras en el transporte y costos de transacción en su comercio, la existencia de cambios estructurales y la presencia de agentes heterogéneos en este mercado. Los resultados sugieren que los modelos lineales pueden no ser los más apropiados para el modelamiento del precio del petróleo.

La no linealidad en el precio del petróleo también fue estudiada por Lixia (2011), quien aplica teoría de sistemas complejos a la serie de precios de futuros diarios del petróleo (no deflactados). A partir de este análisis pronostica precios diarios obteniendo errores de predicción RMSE de 1.431 y MAPE de 1.837. En otro trabajo, Arouri y Nguyen (2010) utilizan técnicas econométricas para analizar la relación de los cambios 
en los precios del petróleo y del gas con el comportamiento de diversas actividades económicas en Europa, encontrando errores en los pronósticos con RMSE entre 1.84 y 1.95 y MAPE entre 0.9817 y 1.2173. También se ha estudiado el efecto del nivel de inventarios de crudo en los países miembros de la OCDE en el pronóstico de sus precios mensuales (Ye et al., 2006). Para tal efecto realizan análisis estadístico de las series de tiempo de inventarios y precios obteniendo resultados satisfactorios con errores RMSE entre 1.7441 y 2.9266 .

Para análisis de corto plazo se encuentran referencias como He et al. (2012) quienes pronosticaron el precio del petróleo aplicando transformada de Wavelet a la serie de precios corrientes diarios (no deflactados) del petróleo WTI y Brent entre los años 2002 y 2011. En otro trabajo, Hou y Suardi (2012) utilizaron 4845 observaciones diarias de los precios corrientes del petróleo WTI y Brent para pronosticar la volatilidad de los retornos. También se tienen modelos de pronóstico semanal usando redes neuronales artificiales (Xiong et al., 2013), donde se toman series de precios corrientes de cierre de semana (no deflactados) con horizontes de predicción hasta de 24 semanas.

Ante el comportamiento no lineal de este tipo de mercados, en la última década se ha incrementado el uso de nuevos métodos basados en redes neuronales artificiales. Su principal característica de permitir establecer relaciones lineales y no lineales entre las entradas y salidas de un sistema ha hecho posible mostrar su aplicabilidad en mercados de alta volatilidad, cuyas variables obedecen a comportamientos no lineales en diversas áreas de la ingeniería y en los mercados energéticos (García et al., 2008; Villada et al., 2011). En la literatura científica se encuentran publicaciones como Haidar et al. (2008), Kuo et al. (2009) y Alizadeh y Mafinezhad (2010); en los cuales se resalta la superioridad en el desempeño de las redes neuronales con respecto a los métodos econométricos y otros modelos lineales. Adicionalmente, se encuentran trabajos que combinan redes neuronales con la lógica difusa. En este sentido, un método híbrido de pronóstico que utiliza redes neuro-difusas e incluye el efecto de cinco indicadores económicos fue propuesto por Azadeh et al. (2012). El modelo mostró ser robusto ante inconsistencias, ruido y no linealidades en los datos; logrando mejor capacidad de generalización que los modelos convencionales.

Dado que la mayoría de referencias consultadas se han enfocado al pronóstico de precios del petróleo en el mediano y largo plazo y que en trabajos previos de nuestro grupo de investigación se ha encontrado un mejor desempeño de los modelos de inteligencia artificial en el pronóstico de precios en los mercados de electricidad, de acciones y de divisas; en este trabajo se propone un modelo basado en redes neuronales para el pronóstico del precio del petróleo WTI en el corto plazo. Se prueban diferentes estructuras de redes neuronales utilizando la serie de precios, el índice del dólar estadounidense y el índice S\&P500 (500 acciones más valiosas negociadas en la bolsa de valores de Nueva York) de seis meses, donde los datos de los primeros cinco se utilizan para entrenamiento dejando el último mes para pronóstico. Los resultados demuestran la aplicabilidad y buen comportamiento de las redes neuronales en mercados energéticos, obteniendo bajos errores en su desempeño tanto dentro como fuera de la muestra.

\section{REDES NEURONALES ARTIFICIALES}

Una red neuronal es un sistema que permite establecer una relación lineal o no lineal entre las salidas y las entradas. Sus características están inspiradas en el sistema nervioso lo que les da varias ventajas tales como su capacidad de aprendizaje adaptativo, son auto-organizativas, pueden funcionar en paralelo en tiempo real y ofrecen tolerancia a fallos por la codificación redundante de la información.

Desde el punto de vista de solucionar problemas, las redes neuronales son diferentes de los computadores convencionales que usan algoritmos secuenciales, mientras que las redes neuronales actúan como el cerebro humano, procesando la información en paralelo, y también pueden aprender y generalizar a situaciones no incluidas en el proceso de entrenamiento. Las redes neuronales pueden procesar información de forma más rápida que los computadores convencionales, pero tienen la desventaja de que no podemos seguir su respuesta paso a paso como se puede hacer al ejecutar un programa convencional en un ordenador por lo que no resulta fácil detectar los errores.

Las redes neuronales artificiales son muy efectivas para resolver problemas complicados de clasificación y reconocimiento de patrones. La más utilizada es la llamada de propagación hacia adelante. La figura 1 muestra una red de propagación hacia delante con dos capas ocultas. El número de entradas es directamente dependiente de la información disponible para ser clasificada mientras que el número de neuronas de salida es igual al número de clases a ser separadas. Las unidades de una capa se conectan unidireccionalmente con las de la siguiente, en general todas con todas, sometiendo a sus salidas a la multiplicación por un peso que es diferente para cada una de las conexiones. 


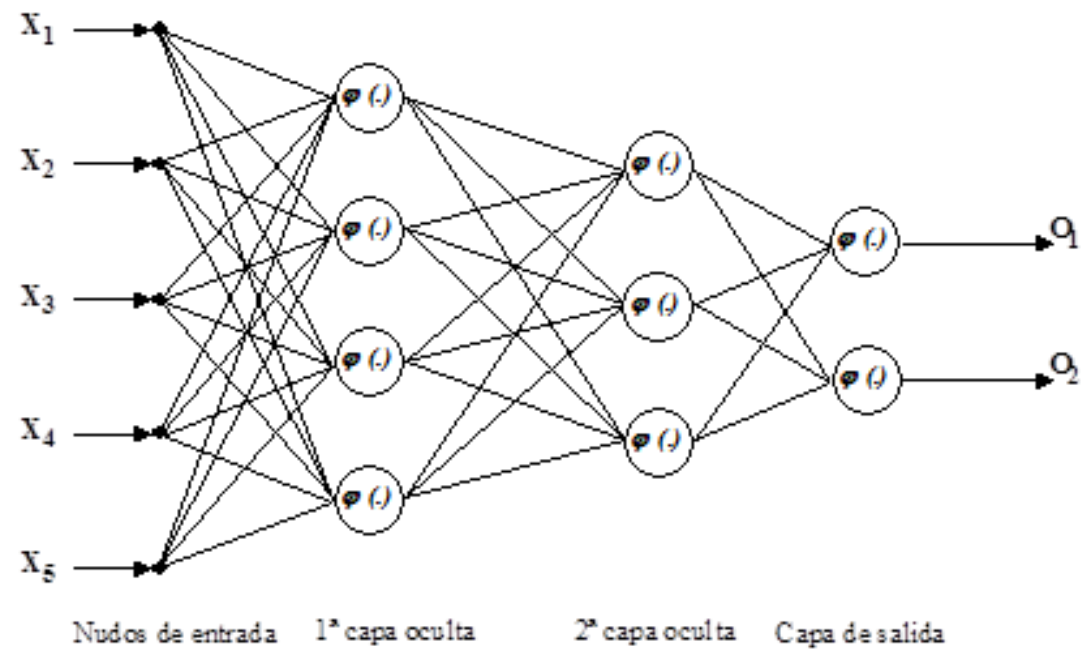

Fig. 1: Red neuronal de propagación hacia adelante

Las Redes Neuronales Artificiales han sido empleadas para resolver numerosos problemas. Entre estos están los económicos y financieros, destacando en gran medida su aplicación en la predicción de series temporales y su capacidad para detectar y explotar la no-linealidad existente en los datos, aun en condiciones donde existen datos incompletos o la presencia de ruido; también se destacan por su desempeño en la solución de problemas complejos, donde el reconocimiento de modelos o comportamientos es importante.

\section{REDES NEURONALES Y PRONÓSTICO DEL PRECIO DEL PETRÓLEO WTI}

Los datos históricos utilizados para el desarrollo del primer modelo fueron obtenidos de la plataforma de Bloomberg (www.bloomberg.com). Las variables de entrada corresponden a los precios de cierre diarios en dólares americanos para el petróleo WTI (West Texas Intermediate) y como salida única se tiene el precio a pronosticar para el día siguiente.

La red que se utilizó fue el perceptrón multicapa con conexiones hacia adelante, porque dentro del marco de las redes de neuronas, el perceptrón ha mostrado ser una de las arquitecturas más útiles en la resolución de este tipo de problemas. Esto es debido, fundamentalmente, a su capacidad como aproximador universal. La arquitectura de esta red, se caracteriza porque tiene sus neuronas agrupadas en capas de diferentes niveles. Cada una de las capas está formada por un conjunto de neuronas y se distinguen tres tipos de capas diferentes: la capa de entrada, la capa de salida y la capa oculta. Cada neurona posee su respectivo nivel de umbral y la función de transferencia utilizada en todas las neuronas fue la tangente hiperbólica.

\section{Algoritmo de Aprendizaje}

La regla o algoritmo de aprendizaje es el mecanismo mediante el cual se van adaptando y modificando todos los parámetros de la red. En el caso del perceptrón multicapa se trata de un algoritmo de aprendizaje supervisado; es decir, la modificación de los parámetros se realiza para que la salida de la red sea lo más próxima posible a la salida proporcionada por el supervisor o salida deseada. Por tanto, el proceso de aprendizaje de la red es equivalente a encontrar un mínimo de la función error. El algoritmo de aprendizaje utilizado fue del tipo Levenberg Marquardt porque en general ha mostrado tener una convergencia más rápida, es decir, requiere menor cantidad de iteraciones para llegar al nivel de error especificado.

\section{Proceso de Aprendizaje}

El objetivo del aprendizaje o entrenamiento de la red, es ajustar los parámetros de la red, pesos y umbrales, con el fin de que las entradas presentadas produzcan las salidas deseadas, es decir con el fin de minimizar la función de error. En lo que respecta al número de capas y neuronas por capa, no existe un método o regla que determine el número óptimo de neuronas ocultas para resolver un problema dado, generalmente se determinan por prueba y error, es decir partiendo de una arquitectura ya entrenada, se realizan cambios aumentando y disminuyendo el número de neuronas ocultas y el número de capas hasta conseguir la arquitectura que se ajuste a la solución del problema. La selección de la mejor estructura en este trabajo se determinó por medio de las medidas tradicionales de evaluación del pronóstico dentro y fuera de la muestra, descritas en la siguiente sección. 
El proceso de entrenamiento es supervisado, el cual consiste en presentar un conjunto correspondiente al $62 \%$ de los datos seleccionados de forma aleatoria y modificar iterativamente los pesos hasta obtener un error mínimo deseado en los resultados. Otro conjunto también aleatorio, con el $20 \%$ de los datos es utilizado para validación permitiendo interrumpir el entrenamiento cuando el error de validación empiece a incrementarse y evitar así el sobre entrenamiento. Finalmente, se prueba la estructura de red obtenida con el $18 \%$ de los datos restantes no incluidos en el proceso de entrenamiento.

\section{Modelo de pronóstico con redes neuronales}

Se probaron diferentes estructuras de redes neuronales con una capa oculta, partiendo de un número de neuronas igual al promedio entre el número de entradas y el número de salidas. Luego se incrementó gradualmente el número de neuronas en dicha capa hasta obtener la estructura más recomendable para el pronóstico del precio de las acciones estudiadas. La selección de la mejor estructura de red, se realiza considerando las siguientes medidas de evaluación dentro y fuera de la muestra: RMSE (Raiz del error medio cuadrático) y el MAPE (Error absoluto porcentual promedio), calculados mediante las ecns. 1 y 2.

$$
\begin{aligned}
& R M S E=\sqrt{\frac{1}{n} \sum_{t=1}^{n}\left(y_{t}^{\prime}-y_{t}\right)^{2}} \\
& M A P E=\frac{100}{n} \sum_{t=1}^{n}\left|\frac{y_{t}^{\prime}-y_{t}}{y_{t}}\right|
\end{aligned}
$$

Donde $n$ es el número de observaciones consideradas, $y_{t}$ es el precio real y $y_{t}^{\prime}$ es el precio estimado por el modelo.

Este trabajo está dirigido a las necesidades de un swing trader operando en una plataforma de negociación virtual, cuyo objetivo fundamental es tener una idea del valor futuro del petróleo WTI en los próximos días; de esta forma se escogió un horizonte de predicción de un mes para el cual se encontró que la red neuronal se entrenaba adecuadamente con los datos de los cinco meses anteriores. En la tabla 1 se presentan los resultados del pronóstico con las medidas de evaluación dentro y fuera de la muestra de las diferentes estructuras de red entrenadas; donde $r$ corresponde al número de rezagos considerados y nn el número de neuronas en la capa oculta.

\begin{tabular}{|c|c|c|c|c|c|c|c|c|c|}
\hline \multirow{3}{*}{$\mathrm{R}$} & \multirow{3}{*}{$\mathrm{nn}$} & \multicolumn{4}{|c|}{$\begin{array}{l}\text { Pronóstico con una variable de entrada (serie } \\
\text { de precios) }\end{array}$} & \multicolumn{4}{|c|}{$\begin{array}{l}\text { Pronóstico con dos variables de entrada (serie de } \\
\text { precios e índice } D X Y \text { ) }\end{array}$} \\
\hline & & \multicolumn{2}{|c|}{ Dentro de la muestra } & \multicolumn{2}{|c|}{ Fuera de la muestra } & \multicolumn{2}{|c|}{ Dentro de la muestra } & \multicolumn{2}{|c|}{ Fuera de la muestra } \\
\hline & & RMSE & MAPE & RMSE & MAPE & RMSE & MAPE & RMSE & MAPE \\
\hline \multirow{4}{*}{1} & 3 & 1.0368 & 0.8435 & 1.0826 & 0.9191 & 1.0231 & 0.8322 & 1.0240 & 0.8338 \\
\hline & 4 & 1.0563 & 0.8477 & 1.0461 & 0.8708 & 0.9745 & 0.8269 & 0.9568 & 0.7995 \\
\hline & 5 & 1.0151 & 0.8308 & 1.0610 & 0.8769 & 0.8830 & 0.7563 & 0.9361 & 0.7660 \\
\hline & 6 & 1.0101 & 0.8417 & 1.0799 & 0.9423 & 0.8754 & 0.7556 & 0.9433 & 0.7632 \\
\hline \multirow{4}{*}{2} & 3 & 1.0306 & 0.8393 & 1.0869 & 0.9600 & 0.8666 & 0.7161 & 0.9470 & 0.8193 \\
\hline & 4 & 1.0193 & 0.7933 & 1.0619 & 0.9072 & 0.9288 & 0.7602 & 1.1437 & 0.8143 \\
\hline & 5 & 0.9142 & 0.7675 & 1.0080 & 0.8454 & 0.7468 & 0.6431 & 1.0673 & 0.8013 \\
\hline & 6 & 0.8844 & 0.7306 & 1.0874 & 0.9467 & 0.7178 & 0.5693 & 1.1452 & 0.8251 \\
\hline \multirow{4}{*}{3} & 3 & 0.9776 & 0.7984 & 1.1144 & 0.9109 & 0.9041 & 0.7580 & 1.0105 & 0.8167 \\
\hline & 4 & 1.0708 & 0.9006 & 1.0091 & 0.8878 & 0.7577 & 0.5982 & 1.0527 & 0.8474 \\
\hline & 5 & 0.9324 & 0.7360 & 1.0248 & 0.9376 & 0.8520 & 0.6731 & 1.0734 & 0.8324 \\
\hline & 6 & 0.9184 & 0.6846 & 1.0670 & 0.9553 & 0.8264 & 0.6766 & 1.0779 & 0.8332 \\
\hline \multirow{4}{*}{4} & 3 & 0.9260 & 0.7616 & 1.0662 & 0.8760 & 0.8522 & 0.7123 & 1.0366 & 0.8611 \\
\hline & 4 & 0.8901 & 0.7314 & 1.1361 & 0.9142 & 0.7350 & 0.6252 & 1.0828 & 0.8543 \\
\hline & 5 & 0.9006 & 0.7356 & 1.0448 & 0.8827 & 0.9181 & 0.7658 & 1.0700 & 0.8943 \\
\hline & 6 & 0.7855 & 0.5945 & 1.0209 & 0.8789 & 0.5067 & 0.4287 & 1.1359 & 0.9287 \\
\hline
\end{tabular}

Tabla 1: Desempeño del valor del petróleo WTI con una y dos variables de entrada 
De un conjunto total de 124 datos de precios de cierre diarios correspondientes a seis meses de negociación comprendidos entre el 13 de diciembre de 2012 y el 6 de junio 2013, se tomaron 102 para entrenamiento (cinco meses) y se dejaron 22 datos correspondientes a un mes completo para pronóstico fuera de la muestra.

Los resultados de la tabla 1 con una variable de entrada muestran en general un buen desempeño de las estructuras de redes estudiadas para modelar el comportamiento del precio del petróleo, en ella, se destacan los indicadores de la estructura 2/5/1 con dos rezagos de tiempo en la capa de entrada y 5 neuronas en la capa oculta donde se tienen unos errores bajos tanto dentro como fuera de la muestra.

Dado el impacto que tiene el crecimiento económico mundial en el precio del petróleo, en la tabla 1 también se involucró en el análisis una segunda variable de entrada que pretende medir este efecto. En este caso se escogió el índice del dólar americano DXY, el cual pondera diariamente el comportamiento del dólar estadounidense frente a una canasta de las seis monedas más importantes (http://investigaciones. bancolombia.com/ espanol/glosariobco/glosarioGrupo2.asp). En la última década se ha podido apreciar que este índice ha mantenido una buena correlación con el desempeño de la economía mundial, depreciándose en épocas de alto crecimiento y apreciándose en temporadas de crisis o incertidumbre, ya que en este último caso los inversionistas liquidan sus activos riesgosos como el petróleo y proceden a buscar refugio en el dólar americano. El agregar el índice del dólar americano como entrada adicional, mejoró de forma apreciable el desempeño de la red tal como era de esperarse al presentar errores más pequeños tanto dentro como fuera de la muestra.

Trabajos recientes han mostrado la relación entre el precio del petróleo y el mercado de valores (Chang y Yu, 2013), por esta razón se probó otro modelo con una segunda variable de entrada que reemplaza el índice del dólar americano por el índice S\&P500 que mide el comportamiento del precio de las acciones de las 500 empresas más grandes de la bolsa de valores de Nueva York. Las columnas del lado izquierdo de la tabla 2 muestran el desempeño de la red con esta nueva variable, con un comportamiento similar a la anterior.

Tabla 2: Desempeño del valor del petróleo WTI con dos y tres variables de entrada

\begin{tabular}{|c|c|c|c|c|c|c|c|c|c|}
\hline \multirow{3}{*}{$\mathrm{R}$} & \multirow{3}{*}{$\mathrm{Nn}$} & \multicolumn{4}{|c|}{$\begin{array}{l}\text { Pronóstico con dos variables de entrada (serie de } \\
\text { precios e índice S\&P500) }\end{array}$} & \multicolumn{4}{|c|}{$\begin{array}{l}\text { Pronóstico con tres variables de entrada (serie de } \\
\text { precios, índice } D X Y \text { e índice S\&P500) }\end{array}$} \\
\hline & & \multicolumn{2}{|c|}{ Dentro de la muestra } & \multicolumn{2}{|c|}{ Fuera de la muestra } & \multicolumn{2}{|c|}{ Dentro de la muestra } & \multicolumn{2}{|c|}{ Fuera de la muestra } \\
\hline & & RMSE & MAPE & RMSE & MAPE & RMSE & MAPE & RMSE & MAPE \\
\hline \multirow{4}{*}{1} & 3 & 1.0381 & 0.8515 & 0.9547 & 0.8104 & 0.9721 & 0.7871 & 1.0999 & 0.8032 \\
\hline & 4 & 1.0108 & 0.8353 & 0.9930 & 0.8102 & 0.9196 & 0.7149 & 0.8701 & 0.7793 \\
\hline & 5 & 0.9863 & 0.7658 & 0.9490 & 0.8008 & 0.9229 & 0.7080 & 0.9828 & 0.7828 \\
\hline & 6 & 0.8605 & 0.6983 & 1.0138 & 0.7646 & 0.8809 & 0.6733 & 0.9725 & 0.7993 \\
\hline \multirow{4}{*}{2} & 3 & 0.9438 & 0.7801 & 1.0343 & 0.9065 & 0.8459 & 0.6616 & 1.1035 & 0.8094 \\
\hline & 4 & 0.9806 & 0.8259 & 0.9566 & 0.8257 & 0.7579 & 0.5861 & 0.9617 & 0.7312 \\
\hline & 5 & 0.8609 & 0.6520 & 1.0808 & 0.9349 & 0.6060 & 0.4633 & 0.9639 & 0.8358 \\
\hline & 6 & 0.5736 & 0.4201 & 0.9583 & 0.8005 & 0.8765 & 0.6414 & 1.0926 & 0.8496 \\
\hline \multirow{4}{*}{3} & 3 & 0.8955 & 0.7265 & 1.1100 & 0.8866 & 0.9123 & 0.7248 & 1.0639 & 0.7929 \\
\hline & 4 & 0.9032 & 0.7196 & 1.0924 & 0.8694 & 0.7771 & 0.5492 & 1.0016 & 0.7361 \\
\hline & 5 & 0.8728 & 0.6602 & 1.0685 & 0.9018 & 1.8164 & 0.6163 & 0.9383 & 0.7846 \\
\hline & 6 & 0.5477 & 0.4462 & 1.1369 & 0.8783 & 0.9251 & 0.7435 & 1.0525 & 0.8108 \\
\hline \multirow{4}{*}{4} & 3 & 0.9914 & 0.7989 & 1.0167 & 0.8396 & 0.8545 & 0.6877 & 0.8620 & 0.7374 \\
\hline & 4 & 0.7399 & 0.6134 & 1.0394 & 0.8468 & 0.6098 & 0.4651 & 0.9762 & 0.7922 \\
\hline & 5 & 0.6189 & 0.5041 & 0.9764 & 0.8193 & 0.9580 & 0.8184 & 1.0835 & 0.7535 \\
\hline & 6 & 0.8565 & 0.6042 & 1.0764 & 0.9138 & 0.8610 & 0.6875 & 1.0042 & 0.7695 \\
\hline
\end{tabular}

Finalmente se probó una estructura de red que incluía tres variables de entrada: la serie de precios, el índice del dólar americano y el índice S\&P500. En las últimas cuatro columnas de la tabla 2 se muestran los resultados para esta nueva estructura, observándose una mejoría en los indicadores de desempeño con respecto a las redes anteriores. Se destaca el mejor desempeño de la red a medida que aumentan los rezagos de tiempo y con cuatro o cinco neuronas en la capa oculta. 


\section{ANÁLISIS DE RESULTADOS}

Las estructuras encontradas de redes neuronales mostraron un buen comportamiento dentro y fuera de la muestra. Al analizar los indicadores de desempeño de las tablas 1 y 2 se encuentra que en general el aumento en el número de rezagos de tiempo mejora el desempeño de la red, pero los mejores valores se obtuvieron para dos rezagos; cuando este número es superior a dos no se percibe una mejora sustancial en las medidas de error. También se aprecia el efecto del incremento del número de neuronas en la capa oculta. Por ejemplo, en la tabla 1 para una variable de entrada y dos rezagos de tiempo, un número de neuronas superior a cinco en esta capa ocasiona una disminución de los indicadores de error dentro de la muestra (RMSE bajó de 0.9142 a 0.8844 ) a la par con un incremento de los mismos fuera de la muestra (RMSE subió de 1.0080 a 1.0874), lo cual indica que la red memoriza cada vez más los datos de entrenamiento pero pierde la capacidad de generalización. Resultados similares se obtuvieron con el error MAPE y con dos y tres variables de entrada.

En forma general se encontró que los menores errores de las tablas 1 y 2 son sustancialmente inferiores a los obtenidos con los modelos de predicción consultados que utilizan los mismos indicadores de desempeño (Arouri y Nguyen, 2010; Lixia, 2011; Ye et al., 2006). En las figuras 2 y 3 se muestra el comportamiento de la red 2/5/1 entrenada con una variable de entrada en la cual se observa que sigue de forma satisfactoria al precio real. La adición de otras variables de entrada como son el índice del dólar americano y el índice S\&P500 mejoraron el desempeño de la red tal como se indicó anteriormente en las tablas 1 y 2. Las figuras 4 y 5 muestran el comportamiento de la red con menores indicadores de error (tres variables de entrada, dos rezagos de tiempo y cuatro neuronas en la capa oculta), donde se aprecia que los pronósticos arrojados son muy próximos a la realidad.

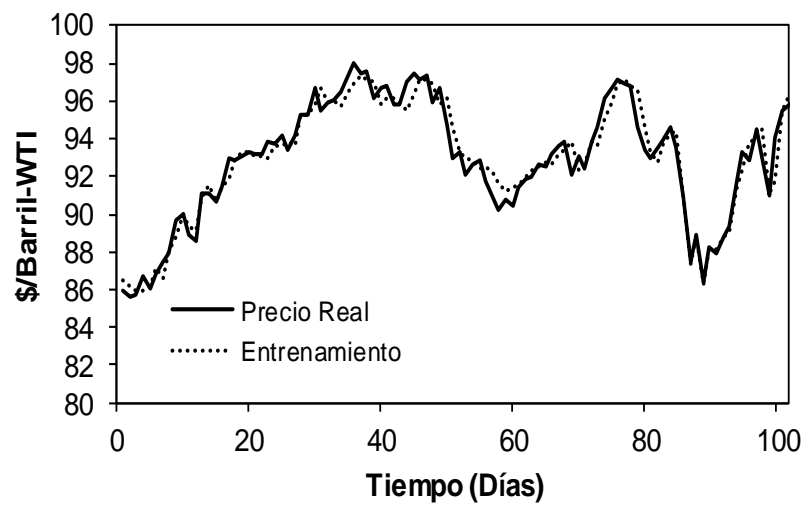

Fig. 2: Pronóstico dentro de la muestra (una variable de entrada)

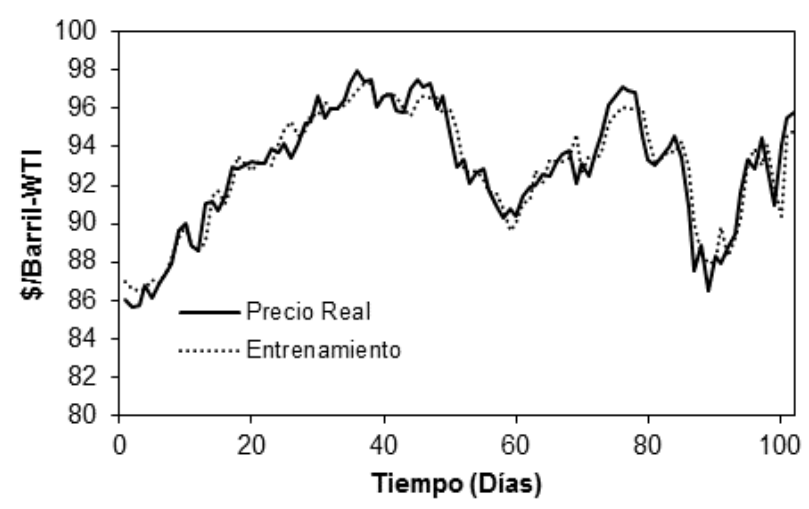

Fig. 4: Pronóstico dentro de la muestra (tres variables de entrada)

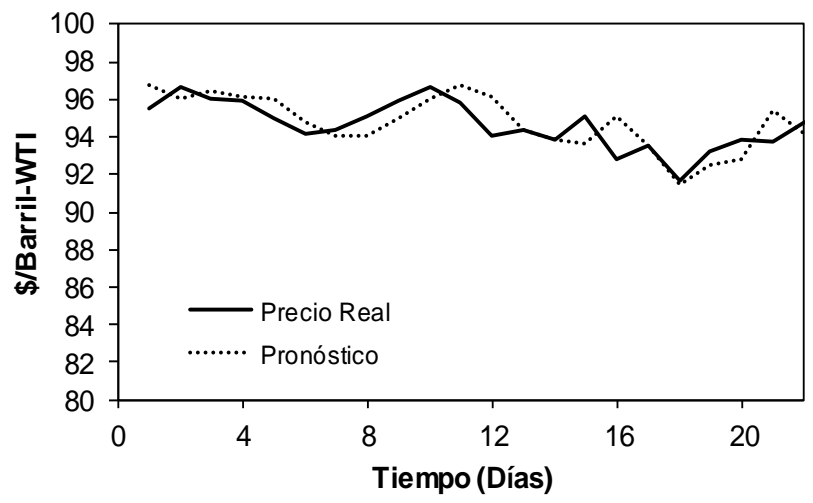

Fig. 3: Pronóstico fuera de la muestra (una variable de entrada)

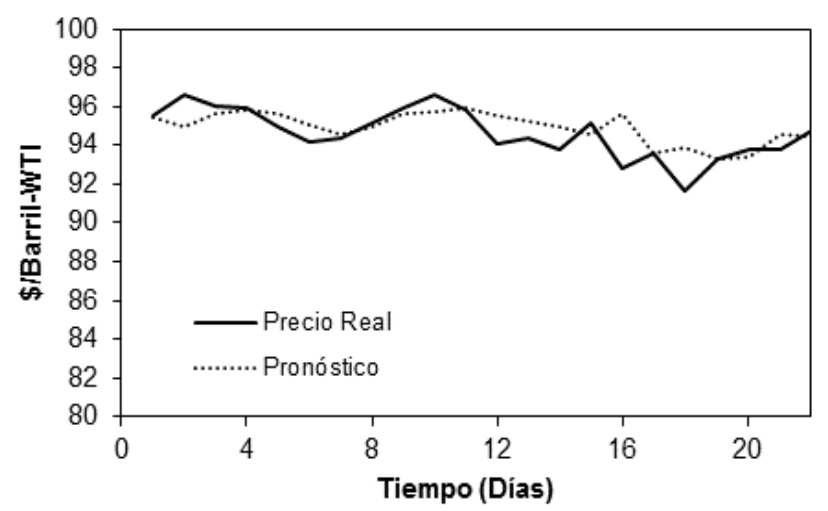

Fig. 5: Pronóstico fuera de la muestra (tres variables de entrada) 


\section{CONCLUSIONES}

Los trabajos previos realizados en nuestro grupo de investigación donde se aplicaron redes neuronales para pronosticar de forma satisfactoria el precio de la electricidad y los buenos resultados de esta investigación en la predicción del precio del petróleo WTI, hacen promisoria su aplicabilidad en otros bienes energéticos. Se concluyen las ventajas de las redes neuronales al ser modelos más sencillos de implementar y permitir obtener bajos errores en el pronóstico tanto dentro como fuera de la muestra.

El efecto de incluir el grado de aversión al riesgo de los inversionistas por medio de los índices DXY y S\&P500, mejoró en forma apreciable el desempeño del modelo. A pesar de que el incremento en el número de rezagos de tiempo y las neuronas en la capa oculta disminuyen de forma gradual el valor de los indicadores de error, se encontró que dos rezagos de tiempo y cuatro neuronas en la capa oculta son suficientes para modelar adecuadamente el comportamiento del precio del petróleo.

\section{AGRADECIMIENTOS}

Los autores agradecen a la Universidad de Antioquia por el apoyo financiero recibido a través del proyecto "Sostenibilidad 2013-2014".

\section{REFERENCIAS}

Alizadeh, A. y K. Mafinezhad, Monthly Brent oil price forecasting using artificial neural networks and a crisis index, Proceedings of the International Conference on Electronics and Information Engineering, Vol. 2, 465468 (2010).

Arouri, M. y D. Nguyen, Oil prices, stock markets and portfolio investment: Evidence from sector analysis in Europe over the last decade, Energy Policy, 38, 4528-4539 (2010).

Azadeh, A., M. Moghaddam, M. Khakzad y V. Ebrahimipour, A flexible neural network-fuzzy mathematical programming algorithm for improvement of oil price estimation and forecasting, Computers \& Industrial Engineering, 62, 421-430 (2012).

Chang, K. L. y S. T. Yu, Does crude oil price play an important role in explaining stock return behavior?, Energy Economics, 39, 159-168 (2013).

Espíndola, C. y J. O. Valderrama, Huella de carbono. Parte 1: conceptos, métodos de estimación y complejidades metodológicas, Revista Información Tecnológica, 23 (1), 163-176 (2012).

Frey, G., M. Manera, A. Markandya y E. Scarpa, Econometric models for oil price forecasting: A critical survey, CESifo Forum, 1, 29-44 (2009).

García, I., A. Marbán, Y. M. Tenorio y J. G. Rodríguez, Pronóstico de la concentración de oxono en Guadalajara-México usando redes neuronales artificiales, Revista Información Tecnológica, 19 (3), 89-96 (2008).

Ghoshray, A. y B. Johnson, Trends in world energy prices, Energy Economics, 32, 1147-1156 (2010).

Haidar, I., S. Kulkarni y H. Pan, Forecasting model for crude oil prices based on artificial neural networks, Proceedings of the 2008 international conference on intelligent sensors, sensor networks and information processing, 103-108 (2008).

He, K., L. Yu y K. Lai, Crude oil price analysis and forecasting using wavelet decomposed ensemble model, Energy, 46, 564-576 (2012).

Hu, A. y S. Suardi, A nonparametric GARCH model of crude oil price return volatility, Energy Economics, 34, 618-626 (2012).

Kisswani, K. M. y S. A. Nusair, Non-linearities in the dynamics of oil prices, Energy Economics, 36, 341-353 (2013).

Kuo, R. J., T. L. Hit y Z. Y. Chen, Evolutionary algorithm-based RBF neural network for oil price forecasting, ICIC Express Letters, 3 (3), 701-705 (2009).

Lixia, L., Nonlinear test and forecasting of petroleum futures prices time series, Energy Procedia, 5, 754-758 (2011). 
Meese, R. y A. Roese, An empirical assesment of non-linearities in models of exchange rate determination, Review of Econometric Studies, 58, 601-619 (1991).

Robles-Agudo, O. y R. Vásquez-Román, Un modelo de programación no-lineal para la planeación de la producción de gas y petróleo, Revista Información Tecnológica, 19 (3), 25-32 (2008).

Villada, F., E. García y J. D. Molina, Pronóstico del precio de la energía eléctrica usando redes neurodifusas, Revista Información Tecnológica, 22 (6), 111-120 (2011).

Xiong, T., Y. Bao y Z. Hu, Beyong one-step-ahead forecasting: Evaluation of alternative multi-step-ahead forecasting models for crude oil prices, Energy Economics, 40, 405-415 (2013).

Ye, M., J. Zyren y J. Shore, Forecasting short-run crude oil price using high- and low-inventory variables, Energy Policy, 34, 2736-2743 (2006).

Zamani, M., An econometrics forecasting model of short term oil spot price, $6^{\text {th }}$ IAEE European Energy Conference, 1-7 (2004). 
